Fractals, Vol. 0, No. 0 (0000)

(c) World Scientific Publishing Company

\title{
SCALING IN COSMIC STRUCTURES
}

\author{
Luciano Pietronero, Maurizio Bottaccio, Marco Montuori \\ Physics Department \& INFM sezione di Roma 1, \\ Università "La Sapienza", P.le A. Moro 2, 00185 Roma, Italy. \\ Francesco Sylos Labini \\ Département de Physique Théorique, Université de Genève, \\ 24, quai E. Ansermet, 1211 Genève
}

\begin{abstract}
Abstract The study of the properties of cosmic structures in the universe is one of the most fascinating subject of the modern cosmology research. Far from being predicted, the large scale structure of the matter distribution is a very recent discovery, which continuosly exhibits new features and issues. We have faced such topic along two directions; from one side we have studied the correlation properties of the cosmic structures, that we have found substantially different from the commonly accepted ones. ¿From the other side, we have studied the statistical properties of the very simplified system, in the attempt to capture the essential ingredients of the formation of the observed strucures.
\end{abstract}

\section{INTRODUCTION}

The existence of a cosmic structures in the universe is one of the most fascinating findings of the two last decades in observational cosmology. Galaxy distribution is far from homogeneous on small scale and large scale structures (filaments and walls) appear to be limited only by the boundary of the sample in which they are detected. There is currently an acute debate on the result of the statistical analysis of large scale features. In the past years we propose a new statistical approach, which has shown surprisingly a fractal structure, extending from small scales to

distance beyond to $40 h^{-1} M p c$ and 
even up to $100 h^{-1} M p c$. In the following we report our findings along two directions of investigations: in the first part we describe the standard analysis and its limits of applicability. We describe our novel analysis and the results we got in the characterization of large scale structures. Contrary to standard claims, this analysis is completely consistent with the results of the standard analysis: it's the interpreation of the latter which is radically different. In the second part, we refer on a study of the dynamics of a very simple model of gravitational formation of structures. We have analysed the evolution and the statistical spatial properties of a $N$-body system of point masses, interacting through gravity. The system is arranged as to simulate an infinite system of particles and with very simple initial conditions.

\section{COSMIC STRUCTURES}

The usual way to investigate the properties of the galaxy spatial clustering is to measure the two point autocorrelation function $\xi(r)^{9,8}$. This is the most used statistical tool, since it can be measured quite accurately with current redshift surveys. $\xi(r)$ is the spatial average of the fluctuations in the galaxy number density at distance $r$, with respect to an homogeneous distribution with the same number of galaxies.

Consider a little volume $\delta V$ at position $\overrightarrow{r_{i}}$; let $n\left(\overrightarrow{r_{i}}\right)$ be the density of galaxies in $\delta V$ and $\langle n\rangle=N / V$ the density of galaxy in the whole sample.

The galaxy density fluctuations in $\delta V$ with respect to the average galaxy density $\langle n\rangle$, i.e. the galaxy relative density fluctuations, is:

$$
\frac{n\left(\vec{r}_{i}\right)-<n>}{<n>}=\frac{\delta n\left(r_{i}\right)}{<n>}
$$

The two point correlation function $\xi(r)$ at the scale $r$ is the spatial average of the product of the relative density fluctuations in two little volumes at distance $r$ :

$$
\xi(r)=<\frac{\delta n\left(\vec{r}_{i}+r\right)}{<n>} \frac{\delta n\left(\vec{r}_{i}\right)}{<n>}>_{i}=\frac{<n\left(\overrightarrow{r_{i}}\right) n\left(\vec{r}_{i}+\vec{r}\right)>_{i}}{<n>^{2}}-1
$$

where the average is performed over the sample. Roughly speaking, a set of points is correlated on scale $r$ if $\xi(r)>0$; it is uncorrelated over a certain scale $R$ if $\xi(r)=0$ for $r>R$. In the latter case the points are evenly distributed at scale $R>r$ or, in another words, they have an homogeneous distribution at scale $R>r$. In the definition of $\xi(r)$, the use of the sample density $\langle n\rangle$ as reference value for the fluctuations of galaxies is the conceptual assumption that the galaxy distribution is homogeneous at the scale of the sample.

Clearly such an approach is valid if the average density $\langle n\rangle$ of the sample is the average density of the distribution, or, in other words, if the distribution is homogeneous on the scale of the sample. For this reason, $\xi(r)$ analysis assumes the homogeneity and it is unreliable for testing it.

In order to use $\xi(r)$ analysis, the density of galaxies in the sample must be a good estimation of the density of the whole distribution of galaxies. This may either be true or not; in any case, it should be checked before applying $\xi(r)$ analysis ${ }^{7}$.

In addition to such criticisms, the usual interpretation of $\xi(r)$ measure is uncorrect for 
spatial distribution of points with respect the amplitude of the $\xi(r)$. The correlation length of the distribution $r_{0}$ is indeed defined as the scale such that $\xi\left(r_{0}\right)=1^{5}$.

Such a definition is uncorrect since, in statistical mechanics, the correlation length of the distribution is defined by how fast the correlations vanish as a function of the scale, i.e. by the functional form of the $\xi(r)$ and not by its amplitude .

The quantity $r_{0}$, then, does not concern the scale of fluctuations and it is not correct referring to this as a measure of the characteristic size of correlations ${ }^{10,11}$. According to the $\xi(r)$ definition, $r_{0}$ simply separates a regime of large fluctuations $\delta n /<n>\gg 1$ from a regime of small fluctuations $\delta n /<n>\ll 1$ : this is correct if the average density of the sample $\langle n\rangle$ is the average density of galaxy distribution .

Such problems of $\xi(r)$ approach can be avoided analysing the spatial correlations of the data set without any a priori assumptions on the homogeneity scale of the data itself. ${ }^{7}$

The way to perform such an unbiased analysis is to study the behaviour of the conditional average number of galaxies $\left\langle N(<r)>\right.$ or the average conditional galaxy density $\Gamma^{*}(r)$ versus the scale $r$. The two quantities are respectively:

$$
<N(<r)>=B \cdot r^{D}
$$

where $N(<r)$ is the number of galaxies contained in a sphere of radius $r$ centered on a galaxy of the sample. $\langle N(r)>$ is the average of $N(<r)$ computed in all the spheres centered on every galaxy of the sample.

$$
\Gamma^{*}(r)=\frac{<N(<r)>}{4 / 3 \pi r^{3}}=\frac{3 B}{4 \pi} \cdot r^{D-3}
$$

$\Gamma^{*}(r)$ is then the corresponding average density of galaxies in spheres of radius $r^{7,12}$ : The exponent $D$ is called the fractal dimension and characterises in a quantitative way how the system fills the space, while the prefactor $B$ depends on the lower cut-off of the distribution.

$<N(<r)>$ and $\Gamma^{*}(r)$ are the suitable statistical tools to detect the two-point correlation properties of a spatial distribution of objects and the possible crossover scale between different statistical distributions.

If the point distribution has a crossover to an homogeneity distribution at scale $R, \Gamma^{*}(r)$ shows a flattening toward a constant value at such scale. In this case, the fractal dimension of Eq.(3) and Eq.(4) has the same value of the dimension of embedding space $d, D=d$ (in three-dimensional space $D=3)^{13,7,12}$.

If this does not happen, the density sample will not correspond to the density of the distribution and it will show correlations up to the sample size. The simplest distribution with such properties is a fractal structure ${ }^{13}$. A fractal consists of a system in which more and more structures appear at smaller and smaller scales and the structures at small scales are similar to the ones at large scales. The distribution is then self-similar. It has a value of $D$ smaller than $d, D<d$. In 3-dimensional space, $d=3$, a fractal has $D<3$ and

$\Gamma^{*}(r)$ is a power law. The value of $N(<r)$ largely fluctuates by both changing the starting point, from which we compute $N(<r)$, and the scale $r$. This is due to the scale invariant feature of a fractal structure, which does not have any characteristic length ${ }^{14,13}$. It is simple to show that if we analyse a fractal structure with $\xi(r)$, we can obtain a value for the correlation length $r_{0}$, which evidently does not have any relation with the correlation properties of the system. In fact such a value is simply a fraction to the size of the sample 
4 Scaling in cosmic structures

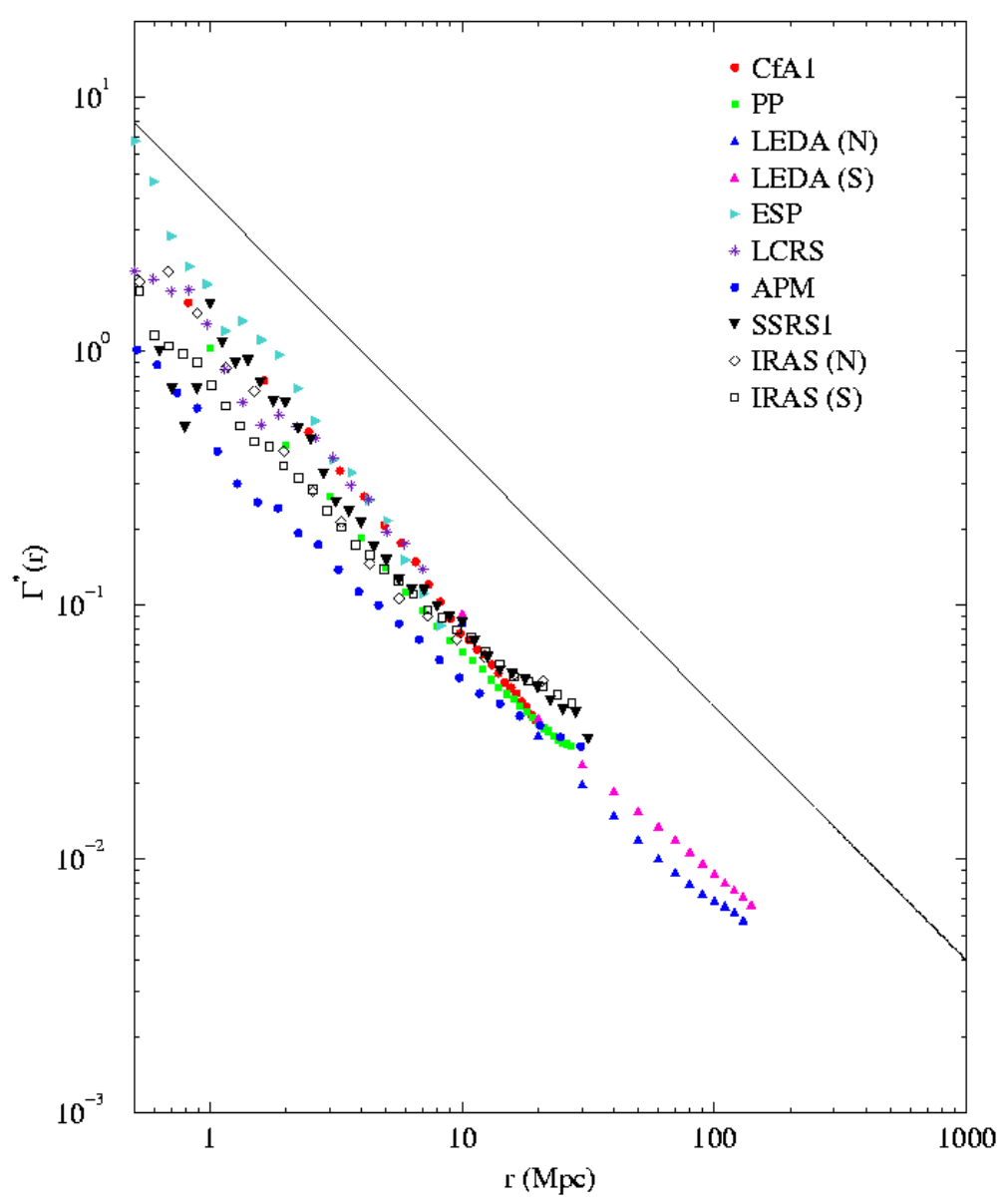

Figure 1: $\Gamma^{*}(r)$ in the range of scales $0.5 \div 100 h^{-1} M p c$ for all the avalaible 3D galaxy data. A reference line with a slope -1 is also shown (i.e. fractal dimension $D=2$ ).

According to our criticism to the standard analysis, we have performed the measure of galaxy conditional average density $\Gamma^{*}(r)$ in all the three dimensional catalogs available. Our analysis is carried out on several 3D galaxy samples. The results are are collected in Fig. $1^{12}$.

$\Gamma^{*}(r)$, measured in different catalogues, is a power law as a function of the scale $r$, extending from $\approx 0.5$ to $30-40 h^{-1} M p c$, without any tendency towards homogenization (flattening) $)^{12}$. In a single case, the LEDA sample ${ }^{16}$, it is possible to reach larger scales, 
smaller scales. This data sample has been largely criticised, but to our knowledge, never in a quantitative way. The statistical tests we performed show clearly that up to $50 h^{-1} M p c$ the results are consistent with all other data ${ }^{12}$.

Such results imply that the $\xi(r)$ analysis is inappropriate as it describes correlations as deviations from an assumed underlaying homogeneity. The galaxy distribution shows instead fractal properties at least in the range $r \approx 0.5-30-40 h^{-1} M p c$, which seem to extend in a single sample up $r \approx 100 h^{-1} M p c$

By consequence, the correlation length $r_{0}$, i.e. the amplitude of the $\xi(r)$, for samples with such a linear extention, should be a fraction of the sample size: $r_{0}$ should be larger for samples whose size is larger.

This is evident in fig. (2), where we plot the results of the standard analysis $\xi(r)$ performed on the same data sets analysed with $\Gamma^{*}(r)$ (fig. (1)).

In this case, then, $r_{0}$ has no relation with the correlation properties of the system; its variation in different samples is not related to any variation of the clustering of the corresponding data set.

\section{SIMULATIONS OF GRAVITATIONAL CLUSTERING}

The study of the formation of the cosmic strucures we have analysed in the above section is one of the most challenging problems in astrophysics. Gravity is the most natural candidate for the explanation of the variety of structures we observe. Indeed, the range of scales on which the gravitational clustering takes place is really impressive: from $10^{-1} p c$ to $10^{8} p c$ $\left(1 p c=3.2615\right.$ light $\left.-y r=3.0856 \times 10^{18} \mathrm{~cm}\right)$. This implies interactions of gravity with other physical processes depending on the scale: from turbulence in cold molecular clouds to cosmological expansion above galaxy cluster scale. Because such a richness of physical processes can be involved in modelling the various structures we observe in cosmos, it is actually very difficult to retrieve a clear picture of the statistical properties of self-gravitating system. Current astrophysical simulations have reached a high level of refinement, both in resolution and in the number of different physical processes which they take into account. Such characteristics allow them to study in great detail the single physical problem for which they are developed ${ }^{4}$. On the other hand they don't allow a clarification the common role and the peculiarities of gravitational interaction. On the contrary, we have tried to focus on such features analysing the most simplest case of a many-body infinite self-gravitating system, without any other ingredient but the gravity. The theoretical approach to such a system goes back to Newton himself ${ }^{1}$, although it has faced by very few authors (e.g. ${ }^{2}$ ). Indeed, the current theoretical effort is quite different since it is devoted to the study of evolution of a continuos gravitating fluid, which is assumed to have peculiar initial density fluctuations ${ }^{5,6}$. ¿From the point of view of statistical mechanics, it is very hard to study the properties of an infinite system of self-gravitating particles. This is mainly due to the long range nature of gravitational potential, which is not shielded by the balance of far away charges, as e.g. in a plasma. Therefore all scales contribute to the potential energy of a particle. The peculiar form of the gravitational potential produces two classes of problems: those due to the short range (i.e. $r \rightarrow 0$ ) divergence and those due to the long range (i.e. $r \rightarrow+\infty$ ) behaviour. The former is not uncommon, since it is the same problem which arises in electromagnetism. The divergence would cause, e.g., the Boltzmann factor to diverge in 


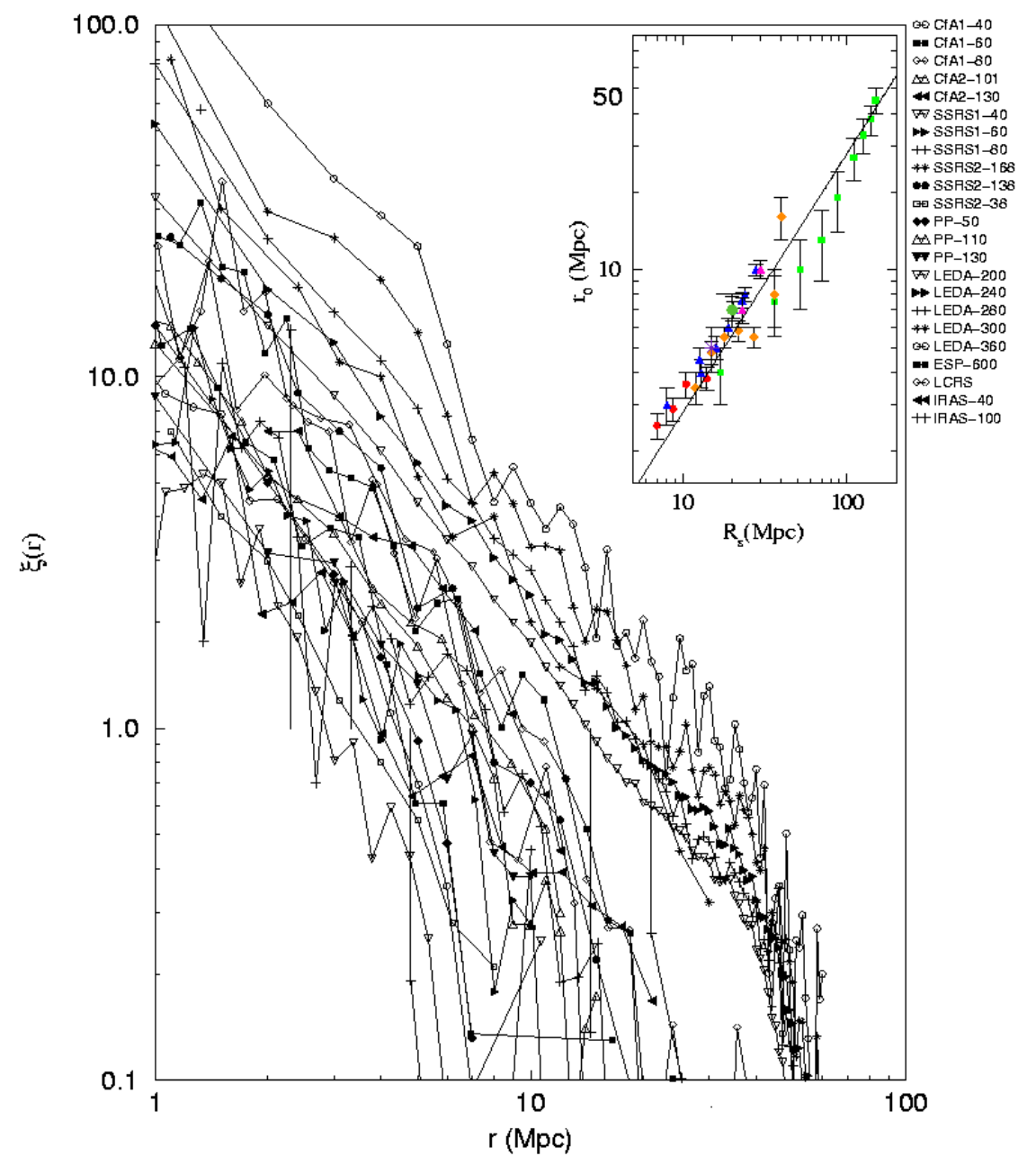

Figure 2: $\xi(r)$ measure in various VL galaxy samples. The general trend is an increase of the $\xi(r)$ amplitude for brighter and deeper samples. In the insert panel we show the dependence of correlation length $r_{0}$ on sample size $R_{s}$ for all samples. The linear behaviour is a consequence of the fractal nature of galaxy distribution in these samples. 
The physical nature of this cut-off may be due to many effects, e.g. the dynamical emergence of angular momentum barriers. The long range behaviour is of much more concern and is, in fact, the problem. It is an easy exercise to verify that the energy of a particle in an infinite self-gravitating system diverges. This causes the energy to be non-extensive. As a consequence, a thermodynamical limit is not achieved, since as the number of particles goes to infinity, even keeping the density constant, the energy per particle diverges. Strangely enough, such a problem has not been fully appreciated by many physicists in the field (see e.g. ${ }^{3}$ ), as they try to avoid the long range divergence by putting the system in a box "as it is usually done with ordinary gas". In fact, the difference is that in ordinary gas, when confining the system in a box, the energy per particle is equal to a constant plus a surface term that goes to zero in the thermodynamical limit. In self-gravitating systems, due to non extensivity, the energy per particle is neither a constant, nor the surface term goes to zero (in fact, it is of the same order of magnitude as the potential energy due to particles belonging to the system).

Another very interesting consequence, which is often not appreciated, is that the thermodynamical definition of temperature, as the parameter which controls the equilibrium of the system, doesn't hold for a self gravitating system, since one cannot divide a system into smaller subsystems with the same thermodynamic properties of the larger system.

As a consequence of such difficulties, a satisfying thermodinamic equilibrium treatment of such systems is still lacking.

However we are much more interested in what happens out of equilibrium, during the evolution of a system.

The system we intend to simulate is a infinite many body system. At this aim, the $N$ particles we effectively consider are confined in a cube of size $L$ submitted to periodic boundary conditions. Every particle in the simulation box interacts with all other particles and with the periodic replicas of the whole system.

The initial conditions we consider are:

(1) random (white noise) initial positions of particles;

(2) no cosmological expansion;

(3) zero initial velocities;

(4) equal mass particles;

Some snapshots of the temporal evolution of the system, with $N=32000$ particles, are shown in Fig. (3). The time evolution goes from the top to the bottom and the initial unclustered distribution of mass points evolves toward a clustered distribution. For each snapshot, we plot on the right, the corresponding $\Gamma^{*}(r)$. A typical time for the evolution of the system is $\tau=1 / \sqrt{G \rho}$, where $G$ is the gravitational constant and $\rho$ is the density of the system. It is roughly the time needed to a particle to cross the system.

Fig. 3 shows some interesting features, that we summarize in the following table.

\section{CONCLUSIONS}

The standard analysis of the correlation properties of the galaxy distribution is performed through the measure of the $\xi(r)$ function. The latter can provide the correct information if the set under analysis is homogeneous inside the sample size. For this reason $\xi(r)$ is not 

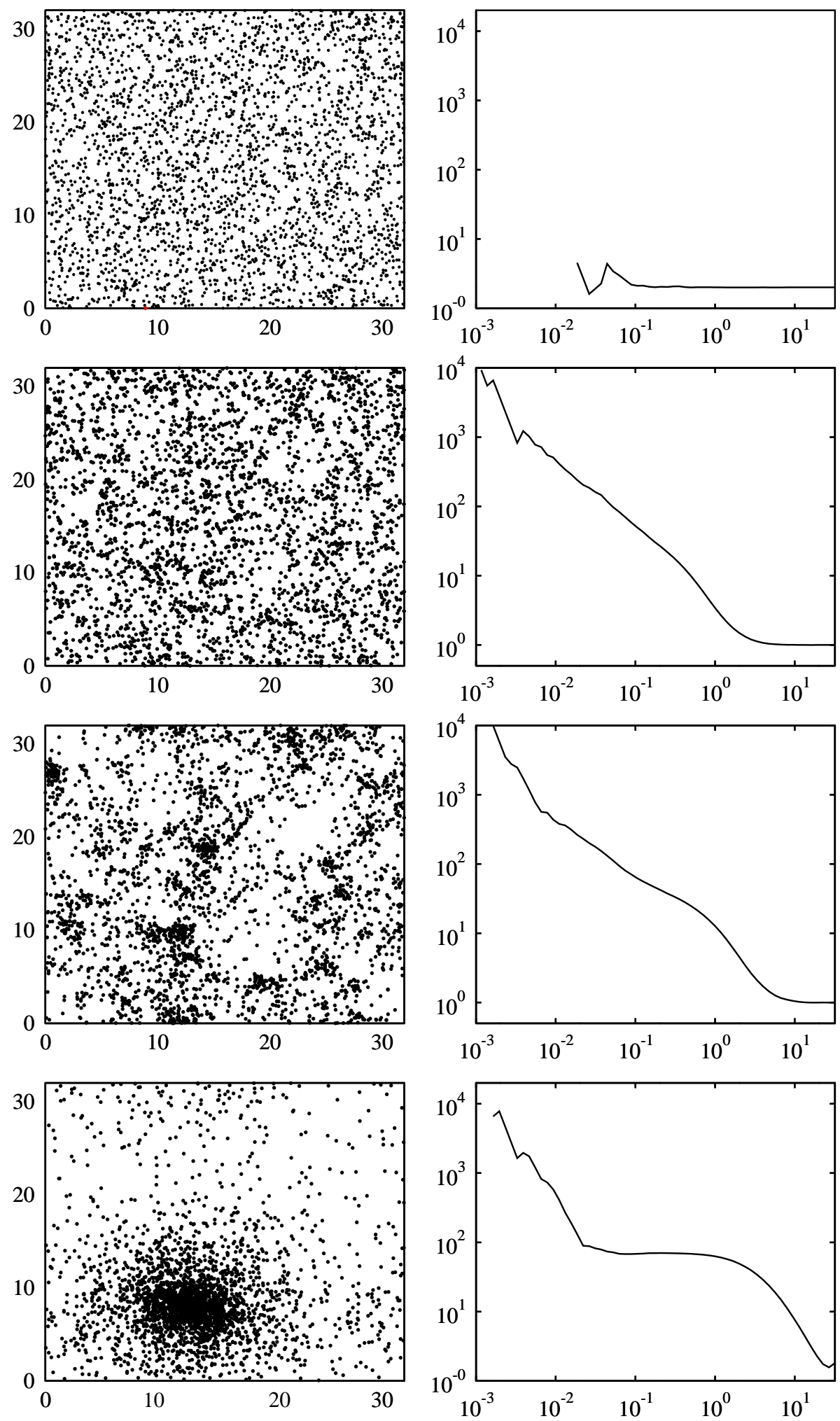

Figure 3: on the left: snapshots of system evolution. The corresponding time is: $t=0$, $t=2 / 3 \tau, t=\tau$ and $t=4 \cdot \tau$ on the right: the corrisponding $\Gamma^{*}(r)$ for the snapshots on the left 


\begin{tabular}{|c|c|c|}
\hline$t$ time & Description & $\Gamma^{*}(r)$ \\
\hline$t_{0}=0$ & $\begin{array}{l}\text { the system is composed by Nparticles } \\
\text { at rest with spatial possonian distribu- } \\
\text { tion in the simulation box }\end{array}$ & $\begin{array}{l}\text { the system has a costant number den- } \\
\text { sity } \Gamma^{*}\left(r, t_{0}\right)=1 \text { at all the scales. } \\
\text { At small scale, } \Gamma^{*}(r) \text { is more fluctuat- } \\
\text { ing, because of the larger poisson noise } \\
1 / \sqrt{N} \text { at small scale }\end{array}$ \\
\hline$t_{1} \approx 2 / 3 \tau$ & $\begin{array}{l}\text { the system starts to cluster at small } \\
\text { scale }\end{array}$ & $\begin{array}{l}\Gamma^{*}\left(r, t_{1}\right) \text { develops a larger amplitude at } \\
\text { small scale }\end{array}$ \\
\hline$t_{2} \approx \tau$ & $\begin{array}{l}\text { the clustering proces evolves with the } \\
\text { merging of the small clusters in bigger } \\
\text { ones }\end{array}$ & $\begin{array}{l}\text { the shape of } \Gamma^{*}\left(r, t_{2}\right) \text { appears to be } \\
\text { quite independent from time, but shifts } \\
\text { toward larger scales for increasing time }\end{array}$ \\
\hline$t_{f} \approx 4 \tau$ & $\begin{array}{l}\text { all the clusters have merged in a sin- } \\
\text { gle big one, with dimension comparable } \\
\text { with the simulation box size }\end{array}$ & $\begin{array}{l}\Gamma^{*}\left(r, t_{f}\right) \text { does not evolve anymore and } \\
\text { the system has reached a stationary } \\
\text { state }\end{array}$ \\
\hline
\end{tabular}

Table 1: on the left: projection of the simulation box onto $x-y$ plane at different time. on the right: corresponding measure of $\Gamma^{*}(r, t)$ at time $t$.

This should be checked before the use of $\xi(r)$ and it is possible through $\Gamma^{*}(r)$ analysis of the sample set. Such an analysis has been performed for the available 3D galaxy samples, with the result that the galaxy distribution appear fractal from $\approx 0.5$ to $100 h^{-1} M p c$.

For such a range of scales, the $\xi(r)$ analysis does not give the correct informations on the statistical properties of the galaxy distribution.

To investigate the formation of such a fractal structure in the universe, we are performing simulations of an N-body infinite self-gravitating system. We started from the simplest initial conditions and analysed the system with the aforementioned $\Gamma^{*}(r)$ function.

Simulations with a different number of particles have shown that the shape of $\Gamma^{*}(r, t)$ for $t \leq \tau$ is independent from $N$. On the contrary, this is not true for the final state of equilibrium, when the system has formed a single cluster ${ }^{15}$.

These measures seems to show that the transient phase,

during which the collapse occurs, posses a well defined

thermodynamical limit, which we are currently analysing ${ }^{15}$.

The discrete nature of the N-body system seems to be a

fundamental ingredient in the development

of the spatial correlations.

The latter, indeed, grow at the small scale, where the discretness

of the point distribution has to be taken into account.

At the moment is not clear if such a system can develop fractal

correlations as seen in the galaxy distribution.

\section{ACKNOWLEDGMENTS}

We would like to thank A.Gabrielli and M.Joyce for enlighting discussions. We acknowledge financial support from EC TMR Research Network under contract ERBFMRXCT960062 
Scaling in cosmic structures

\section{REFERENCES}

1. I.Newton, quoted in Theories of the Universe ed. M. K. Munitz (Toronto, 1957), p. 211.

2. W. C. Saslaw The Distribution of the galaxies (Cambridge University Press, 2000).

3. T.Padmanabhan Phys. Rep. 188, 285 (1990);

4. for references to astrophysical $N$-body simulations see: http : //star - www.dur.ac.uk/ frazerp/virgo/virgo.html http ://star - www.dur.ac.uk/ cole/mocks/main.html http ://lca.ncsa.uiuc.edu : 8080/GC3 ${ }_{H}$ ome ${ }_{P}$ age.html

5. P. J. E. Peebles Principles of Physical Cosmology (Princeton University Press, 1993).

6. T. Padmanabhan Structure formation in the universe (Cambridge University Press, 1993).

7. P.Coleman \& L.Pietronero Phys. Rep. 213, 311 (1992);

8. P. J. E. Peebles Ap.J. 185, 413 (1973);

9. H.Totsuji and T.Kihara Pub.of Astron. Soc. Jap. 21, 221 (1969);

10. J. Gaite, Domnguez A. and Prez-Mercader J. Ap. J.Lett. 522, L5 (1999);

11. A. Gabrielli, F. Sylos Labini, R. Durrer Ap. J.Lett. 531, L1 (2000);

12. F. Sylos Labini, M. Montuori, L. Pietronero 1998 Phys. Rep. 293, 66 (1998);

13. B. Mandelbrot, The Fractal Geometry of Nature (Freeman, San Francisco, 1983);

14. M. Montuori, F. Sylos Labini, A. Gabrielli, A. Amici and L. Pietronero Europhys. Lett. 39, 103 (1997)

15. M.Bottaccio, A.Amici, P.Miocchi, R. Capuzzo Dolcetta, M. Montuori and L. Pietronero Phys.Rev.Lett. submitted (2001)

16. Di Nella H. et al. A\&A Lett. 308 L33 (1996) 